\title{
1 Migratory birds as global dispersal vectors
}

2 Duarte S. Viana ${ }^{1 *}$, Luis Santamaría ${ }^{2}$ and Jordi Figuerola ${ }^{3,4}$

3

$4 \quad{ }^{*}$ Corresponding author: Viana, D.S. (dviana@ebd.csic.es)

$5{ }^{1}$ Estación Biológica de Doñana, CSIC, C/Américo Vespucio, Sevilla, s/n, E-41092,

$6 \quad$ Spain. E-mail: dviana@ebd.csic.es

$7 \quad{ }^{2}$ Estación Biológica de Doñana, CSIC, C/Américo Vespucio, Sevilla, s/n, E-41092,

$8 \quad$ Spain. E-mail: luis.santamaria@ebd.csic.es

$9{ }^{3}$ Estación Biológica de Doñana, CSIC, C/Américo Vespucio, Sevilla, s/n, E-41092,

10 Spain. E-mail: jordi@ebd.csic.es

$11{ }^{4}$ CIBER Epidemiología y Salud Pública (CIBERESP), Sevilla, Spain

12

13 Keywords: migratory birds, long distance dispersal, propagule dispersal, range shifts, 14 biodiversity distribution. 


\section{Abstract}

16 Propagule dispersal beyond local scales has been considered rare and unpredictable.

17 However, for many plants, invertebrates and microbes dispersed by birds, long distance

18 dispersal (LDD) might be regularly achieved when mediated by migratory movements.

19 Because LDD operates over spatial extents spanning hundreds to thousands of

20 kilometers, it can promote rapid range shifts and determine species distributions. We

21 review evidence supporting this widespread LDD service and propose a conceptual

22 framework for estimating LDD by migratory birds. Although further research and

23 validation efforts are still needed, we show that current knowledge can be used to make

24 more realistic estimations of LDD mediated by regular bird migrations, thus refining

25 current predictions of its ecological and evolutionary consequences.

27 The need to quantify long distance dispersal

28 Long distance dispersal (LDD) allows organisms to cross population boundaries, move

29 among habitat patches and colonize remote areas, thus having important ecological,

30 biogeographical and evolutionary consequences [1-3]. Its study and quantification has

31 been, however, hindered by the low frequency of LDD events, the difficulty of tracking

32 propagules (see Glossary) over large geographic scales, and the unpredictable nature of

33 LDD vectors operating at such scales (such as ocean currents, extreme meteorological

34 events and animals moving over long distances) [4].

Animal vectors are diverse and provide LDD services in a wide range of

36 ecosystems and biogeographic regions. Examples of animal vectors able to disperse

37 seeds over distances of hundreds to thousands of meters include Amazonian fish $(<5$

$38 \mathrm{~km})$ [5], Asian elephants (3 up to $5.8 \mathrm{~km})$ [6], North American deer $(<3 \mathrm{~km})$ [7] and

39 African hornbills $(<6.9 \mathrm{~km})[8]$. Fruit bats and pigeons are also known to disperse seeds 
of many plant species over tens of kilometers throughout the tropics and in some subtropical regions $[9,10]$. But among animal vectors, birds have the highest potential to mediate propagule LDD, especially during migration $(>1,000 \mathrm{~km})[11]$. However, dispersal is hard to measure and quantify, especially LDD events. Therefore, the study of biodiversity distribution has been hindered by a deficient understanding and incorporation of dispersal, namely through the use of theoretical and arbitrary dispersal kernels. The most popular example is species distributions models (SDMs), which either disregard dispersal or incorporate very crude formulations of dispersal kernels (e.g. $[12,13])$. Moreover, many studies on phylo- and bio-geographic patterns (reviewed in $[2,14]$ ) argue that LDD promoted by birds is the only parsimonious explanation for such patterns in many taxa, including angiosperms $[15$, 16], bryophytes $[17,18]$, freshwater zooplankton $[19,20]$, marine snails [21] and ticks $[22]$.

The potential of birds to mediate LDD of a vast number and diversity of organisms (see Box 1) provides a solid conceptual and methodological background to study vectored LDD and progress towards its quantification. Albeit still limited by technological and methodological constraints, progress so far allows for much better LDD estimations than before. We review the vectoring role of birds, especially of migratory birds, and propose an improved conceptual framework for understanding and estimating bird-mediated LDD beyond the scale of local populations.

\section{Overlooked vectoring potential of migratory birds}

Birds are probably the most abundant and competent vertebrate vectors [23]. They can disperse propagules both internally, following voluntary or involuntary ingestion of propagules (endozoochory), and externally, following attachment of propagules to 
65 feathers or legs. Birds also transport entire organisms, including pathogens and

66 parasites, in both ways $[24,25]$ (Box 1).

Among birds, migratory species can be key LDD vectors because: (i) they move seasonally over broad spatial scales and can overcome major geographical barriers; (ii)

they stop at sites with similar habitat characteristics along their migration routes,

70 increasing the probability of successful establishment of dispersed propagules (i.e. they

71 provide directed dispersal); and (iii) they are diverse, abundant and ubiquitous. Nearly one fifth (19\%) of the 10,064 extant bird species on Earth (BirdLife International) are fully migratory [26] and many other species make long-distance movements (such as

74 altitudinal or irruptive movements) as well as dispersal movements. Although migratory

75 birds occur all over the world, the vast majority occurs in higher latitudes, especially in

76 the northern hemisphere [26]. This means that LDD by migratory birds can be expected

77 to be more frequent and relevant in the temperate region of the northern hemisphere,

78 although the role of altitudinal, intra-tropical, temperate-tropical (e.g. by frugivorous

79 songbirds [27]) and trans-hemispheric (e.g. by waders [28]) migrations should not be 80 neglected.

81 Quantifying LDD by birds engaged in long-distance movements is a daunting

82 task, as propagules must be sampled while the bird is in flight or immediately after

83 stopping, but increasing evidence provides convincing support for this phenomenon.

84 For example, $1.2 \%$ of passerine and gallinaceous birds intercepted by falcons while 85 migrating over the ocean from Europe to Africa were found to transport ingested seeds 86 (endozoochory) of at least five plant species [29]; and eight species of trans-equatorial 87 migrant waders, captured in their arctic breeding grounds shortly before migration, were 88 found to have bryophyte diaspores attached to their plumage, suggesting that these birds 89 transport plant propagules towards their wintering grounds [30]. Numerous studies of 
seed dispersal to and between oceanic islands also suggest that marine and migratory birds are important LDD vectors (see review in [31]). The most striking example comes from Surtsey Island, a volcanic island nearby Iceland whose flora is dominated by birddispersed angiosperms (64\% of species [31]), and where a single passerine species

94 arriving from migration was found to carry seeds of 30 different plant species [32].

95 Dispersal of parasites and pathogens during bird migration also provides illustrative 96 examples. Molecular analysis showed that $0.2 \%$ of the migrating birds sampled in an offshore island of New England were infested with ticks originating from coastal Maine (9.7 km away), $20 \%$ of which were infected with Lyme disease, a pathogen that was absent from the island [22]. Similarly, the spread of West Nile Virus across North

100 America and the transcontinental spread of avian Influenza were mediated by migratory songbirds and migratory waterfowl, respectively [24].

As expected, LDD by migratory birds seems to be more frequent in the temperate region of the northern hemisphere. However, this bias might also reflect the

104 larger number of studies undertaken in this region. In the tropical, subtropical and 105 southern-temperate regions, many bird species fly long distances within short time 106 periods, during both migration and other long-distance displacements. Examples of suitable long-distance dispersal vectors from these regions include hornbills in tropical Africa $(<290 \mathrm{~km})[8]$, oilbirds and pigeons in South America $(>100 \mathrm{~km})[33,34]$, witheyes, bulbuls and mousebirds in South Africa $(<400 \mathrm{~km})$ [35], waterfowl in Australia 110 (hundreds of kilometers) [36] and gulls all over the world (hundreds of kilometers to 111 and between oceanic islands) [31]. Although all the aforementioned studies are of key importance to establish the

113 likelihood and scale of LDD by birds, they are not suited for estimating realistic 114 dispersal patterns (e.g. dispersal kernels), due to their opportunistic nature (only a 
115 handful of species and localities available), low sample sizes and limited spatial

116 accuracy in the determination of source populations. Moreover, propagules from each

117 different vectored species may be dispersed by a diverse guild of vectors, each of them

118 with different vectoring capacities, adding a level of complexity to the use of

119 observational studies to understand vectored LDD. To overcome these limitations,

120 mechanistic (process-based) models can be used to estimate potential LDD [4]. Despite

121 recent methodological progress in estimating dispersal of organisms transported by

122 migratory birds (e.g. [11, 37]), the lack of a unified conceptual framework has hindered

123 the achievement of more realistic estimations and predictions to date.

\section{A framework for the study of LDD by migratory birds}

126 Propagule dispersal comprises three consecutive phases: initiation (propagule uptake by

127 the vector), transport (propagule movement along with the vector) and deposition

128 (propagule retrieval following transport) [4]. To understand the various determinants of

129 each of these three phases, it is particularly useful to consider the Movement Ecology

130 Framework proposed by Nathan et al. (2008) [38], which comprises four basic

131 components: internal state, motion capacity, navigation capacity and external factors.

132 Below we build on this conceptual framework to provide a mechanistic model of

133 propagule movement mediated by migratory birds (see the conceptual model in Figure

134 1). Because propagule movement is mediated by the vector, the movement ecology of

135 the vectored organism should be regarded as nested within the movement ecology of the

136 bird vector [10]. This general framework can be applied to all kinds of propagules,

137 though there are obvious differences among them (e.g. diaspores vs. parasites) that are

138 not extensively reviewed here. For example, most parasites and pathogens, but not other

139 propagules, can (i) influence the vector's behavior, movement and dispersal capacity, 
140 especially if disease is involved, and (ii) propagate while retained in the vector, thus

141 increasing their dispersal effectiveness.

\section{Propagule uptake}

144 The dispersal process initiates when the vector acquires the propagule. Hence, it is

145 contingent upon the biotic interaction between the vector (in this case the migratory

146 bird) and the vectored organism (through its propagules) - thus, on their spatial,

147 temporal and ecological overlap. Phenological synchrony between propagule production

148 and vector visitation has been observed in several regions and biomes. For example,

149 many terrestrial and aquatic plants produce their fruits during the autumn migration of

150 frugivores and waterbirds, respectively $[39,40]$. Further, the odds of acquiring parasites

151 and pathogens are expected to be high during migration, because migratory birds are

152 known to congregate in great numbers in key stopover areas along flyways. The

153 probability of encounter between vectors and propagules represents the "navigational"

154 capacity of the vectored organisms and is determined, for instance, by propagule traits

155 that attract the dispersal vector and/or allow propagule uptake (e.g. production of fleshy

156 fruits promoting ingestion, adhesive structures promoting attachment, and air- or vector-

157 borne disease propagules promoting transmission) $[10,41]$.

158

Overall, the initiation phase is driven by (i) the internal state of the vector,

159 namely its necessity to replenish energy for migratory flights [42], which determines the

160 identity and quantity of acquired propagules; and (ii) the internal state and navigation

161 capacity of the vectored organisms, which determine the characteristics, phenology

162 (time of production) and abundance of their propagules. External factors can also affect

163 the initiation phase: for example, climatic conditions can influence propagule 
164 production, attractiveness and availability, while meteorological conditions can

165 influence migration time and stopover use by birds.

166

\section{Transport: bird movement}

168 Following the initiation phase, migratory birds start or resume migration (see Box 2)

169 and transport internal and/or attached propagules. The duration and distance of the

170 migratory flight depend on the birds' navigational and motion capacities, particularly on

171 the trade-off between energy consumption and total migration time. This trade-off

172 forms the basis of the "optimal migration" theory $[42,43]$ and defines the different

173 (optimal) migratory strategies observed amongst different bird species, which in turn

174 determine propagule LDD patterns [44].

175 From the vectored organism's perspective, its "motion" capacity depends on: (i)

176 the retention time of propagules, which is determined by a number of propagule traits

177 (notably size; e.g. [45], but also presence of specialized structures [46]); (ii) their

178 resistance to the aggression encountered in the bird's body (gut environment and

179 immune responses, for internal dispersal), or to the environmental conditions at the

180 vector's exterior while in movement. External factors, such as landscape configuration

181 and weather conditions, affect vector (and thus propagule) movement by shaping its

182 movement decisions and route $[47,48]$.

184 Propagule deposition

185 Finally, propagules are released and deposited, either during flight, probably resulting in 186 establishment failure, or after the bird stops, often in a habitat type comparable to that of 187 departure, thus increasing the chances of propagule successful establishment. Stopping 188 over during migration depends on the navigational capacity of the bird, i.e. on its ability 
to find shelter and food en route, and its internal state (willingness to stop). The

190 deposition of viable propagules depends on their resistance to the internal or external

191 conditions experienced during transport and their retention time (see Box 3).

192 Germination, hatching and/or transmission of transported propagules depend on the

193 effects of the conditions endured during transport and the propagule's internal state, as

194 determined by the life-history of the species and modulated by propagule traits (e.g.

195 coat permeability and presence of dormancies) and environmental cues (e.g.

196 photoperiod and temperature). External factors such as habitat characteristics will also

197 determine the fate of retrieved propagules.

198

199 Effectiveness of LDD

200 The realization of dispersal depends on its effectiveness, i.e. on the combination of

201 successful transportation and deposition of viable propagules, plus their successful

202 establishment and reproduction. Such effectiveness is critically related to the gains and

203 costs involved in reaching distant habitat patches through LDD (e.g. [49]), and

204 ultimately depends on the constraints posed by a combination of abiotic and biotic

205 filtering of arriving propagules. The expected establishment challenges further increase 206 uncertainty to the whole LDD process.

207 Dispersal effectiveness can be measured by the product of the number of 208 propagules dispersed by a vector and the probability that they produce a new adult (i.e.,

209 by the quantity and quality components of dispersal) [50]. Field studies in aquatic

210 ecosystems report high prevalence of propagules in waterbird droppings ( $45 \%$ for

211 aquatic plants and $32 \%$ for invertebrates, on average), with high germination or

212 hatching potential (36\% and 30\%, respectively) [51]. Terrestrial birds also ingest and

213 disperse large amounts of propagules, especially seeds, during their migration [27, 52, 
214 53]. Many of the seeds defecated by frugivorous birds remain viable after

215 transportation, and most show enhanced (36-41\%) or unaffected (45-48\%) germination

216 frequency and rate $(\mathrm{N}=153$ and 103 plant species for germination frequency and rate,

217 respectively) [54]. These numbers are all the more important if one considers the large

218 population numbers of bird vectors: e.g. two migratory bird species, one waterfowl

219 (mallard) and one passerine (European pied-flycatcher) known to ingest large quantities

220 of propagules during migration [51,52], have a worldwide population which surpasses

22119 and 40 million birds, respectively (according to BirdLife International). Therefore,

222 these birds alone likely disperse hundreds of thousands to millions of viable propagules

223 each year. Passerines are generally more abundant than waterbirds, but the latter can

224 acquire larger propagule loads, make longer migratory flights (Box 2) and retain

225 propagules over longer periods (Box 3); thus the amount of propagules that reach a

226 given distance is expected to depend on a tradeoff between the number of vectors

227 (which generally decreases with body size; [55]) and their motion and propagule

228 retention capacities (which generally increases with body size; see Box 2 and 3).

229 Successful colonization and establishment in the destiny will ultimately depend on

230 niche processes. As such, LDD might be more effective in aquatic ecosystems because

231 waterbirds are more likely to fly from and to waterbodies - which are relatively

232 homogeneous habitats. Indeed, the broad distribution of many aquatic organisms has

233 been often attributed to the relative homogeneity of the aquatic environment (see [56]

234 for a discussion). Nevertheless, recruitment probabilities in general may increase

235 through phenotypic plasticity [56], rapid adaptation to local conditions [57], and

236 directed local-scale dispersal to suitable microhabitats [58].

\section{Ecological consequences of LDD}


239 Migratory birds can promote the movement and connectivity of many taxa over

240 extremely large spatial scales, with important ecological consequences. They can

241 promote large-scale connectivity in anthropogenic (e.g. forest-pasture mosaics) and

242 naturally isolated (e.g. lakes and wetlands, mountain tops) landscapes [59, 60]; as well

243 as the colonization of distant habitat patches, including those in different continents [24,

$24461]$ or hemispheres [16, 17], and on oceanic islands [29, 62], hence contributing to the

245 formation of phylo- and biogeographic patterns. LDD can also accelerate the spread of

246 biological invasions [63, 64], parasites and pathogens [22, 24], and is likely to mediate

247 the responses of species and populations to global change [64-66].

248

249 Estimation of ecological consequences: rapid range shifts

250 LDD is predicted to accelerate greatly the rate of dispersal across large spatial extents.

251 However, and despite the wide acknowledgement of its importance in modern modeling

252 platforms (e.g. $[12,13,67])$, the dispersal component of current species distribution

253 models (SDMs) remains poorly defined. In most cases, it assumes either unlimited

254 dispersal or an arbitrary dispersal kernel applied across all species. In the few studies

255 that include dispersal kernels estimated for specific species, such estimates do not

256 contemplate the role of LDD by non-standard vectors such as migratory birds (e.g.

257 [68]). We argue that the conceptual framework presented here, together with the

258 increasing amount of published evidence, may allow for the incorporation of more

259 realistic predictions of the frequency and scale of LDD provided by migratory birds to a

260 considerable number of species - albeit accurate predictions of the distance and

261 direction of LDD events will only be attainable if both bird movement and propagule

262 retention time are accurately parameterized (see Box 2 and 3). 
In Figure 2, we illustrate how to estimate and predict rapid range shifts for

264 species dispersed by migratory birds, based on the conceptual framework presented

265 above. This example can constitute a methodological basis to foster the incorporation of

266 LDD potential in species distribution modeling. For a given species and/or population

267 distributed over a given area and dispersed by a given set of migratory bird species, we

268 estimate its possible range shift within one year (one spring and one autumn migration).

269 The core model component is the dispersal kernel, which was estimated according to a

270 mechanistic model [11]. Bird migratory-flight distances (see Box 2) are combined with

271 propagule retention times (see Box 3) to produce the dispersal kernel. Note that, if the

272 model is to be parameterized for pathogen dispersal, the effect of the infection (i.e.,

273 propagule retention) on the migration capacity of vector birds should be adequately

274 incorporated (see Box 3 and references therein).

275 Once the dispersal kernel is estimated, habitat suitability along the migration

276 flyway must be determined to estimate the combined probability of propagule arrival

277 and establishment in a given locality. Habitat suitability might be estimated through

278 niche modeling, incorporating whenever possible the interaction between abiotic, biotic

279 and stochastic population and community factors. The example in Figure 2 provides the

280 possible range shifts of a vectored population across a full migratory cycle (one spring

281 and autumn migration), which may be easily run over multiple years. If the goal is to

282 predict future range shifts (e.g. following climate change), stepping-stone LDD events

283 should be included by complementing these models with demographic models

284 predicting propagule production at each new site of establishment (e.g. [67]). 
287 LDD predictions might be tested using a combination of direct observations and 288 analysis of their ecological consequences. Direct observations of LDD (e.g. [29]) might 289 be achieved by examining birds arriving from long-distance flights, such as those killed 290 while in active migration by predators, human hunters or collision with man-made 291 structures (e.g. lighthouses or wind turbines). The origin of collected propagules might 292 then be traced using stable isotopes or genetic markers (see [69] for a review). For 293 example, LDD frequencies observed empirically in one study $(1.2 \%$ of the sampled 294 migrating birds were transporting at least one propagule [29]) is comparable with 295 mechanistic-model estimates (yielding LDD frequencies of $\leq 3.5 \%$ of the migrating 296 birds [11]).

297 Ecological consequences, namely distributional patterns, can be investigated 298 using taxonomic assessments, phylogenetic analyses, genomic analyses, niche 299 modeling, and computational techniques for modeling evolutionary data (see [2] for an overview). Inference made from distributional patterns might be used to validate LDD

301 predictions. For example, it has been shown that the distribution of aquatic and land 302 angiosperms [70, 71], zooplankton [19, 20], and pathogens [24] can be explained by 303 regular dispersal along the migratory routes of their potential bird vectors. While regular 304 LDD might take place at ecological time scales, providing a feasible response 305 mechanism to rapid environmental changes such as climate change, rare events that 306 promote the colonization of remote areas and generate disjunct distributions, such as 307 bipolar distributions (e.g. [16]), might take place at evolutionary time scales [2], posing 308 insurmountable challenges to the possibility of predicting their occurrence.

\section{Concluding remarks and future directions}


311 A wide range of organisms uses the LDD services provided by birds; hence more

312 accurate LDD estimations might be achieved by incorporating the birds' vectoring

313 potential and thus the full dispersal potential of vectored organisms. The study of

314 diaspore (e.g. seed) dispersal and pathogen dispersal have traditionally been studied in

315 parallel research lines, but studying the common and distinct processes underlying their

316 dispersal might contribute to and cross-fertilize both research lines. The proposed

317 framework constitutes a first step towards a general mechanistic understanding of bird318 mediated LDD.

319 Although data is still limited for many vector and vectored species, LDD

320 estimations based on mechanistic models and allometric relationships (see Box 4)

321 provide more reliable estimates than the most commonly assumed dispersal scenarios

322 (of unlimited or arbitrary dispersal capacity). Our ability to quantify and predict LDD

323 by migratory birds will critically depend on the effectiveness of dispersal: (i) LDD

324 might be more predictable if propagules are frequently acquired along migratory routes

325 (e.g. [24, 29, 41, 52, 72]), and (ii) LDD might be largely unpredictable whenever

326 propagule transportation occurs at very low frequency, especially in the case of extreme

327 events spanning very large distances (hundreds to thousands of kilometers; e.g. [15,

328 16]). Movement tracking technology is expected to boost research on species range

329 dynamics that will contribute to understand global patterns of biodiversity [72].

330 The conceptual framework proposed here can be used to derive and test specific

331 hypotheses about the effects of LDD on (i) colonization patterns and connectivity, and

332 consequent biogeographic patterns, and (ii) the spread of parasites, pathogens and

333 invasive species. Reliable estimations of LDD will aid in (1) improving species

334 distribution models (SDMs), by indicating where and when species, including invaders

335 and disease, can reach suitable habitat patches, (2) choosing adequate scales to survey 
336 the distribution of biodiversity (e.g. spatial and temporal turnover in local

337 communities), and (3) predicting species responses to global change. Therefore, it will

338 have clear implications for the conservation of biological diversity and the sustainable

339 use of ecosystem services.

340

341 Box 1. Diversity and LDD potential of organisms dispersed by birds

342 A wide array of different taxa use the LDD services provided by birds. Microorganisms,

343 including viruses, bacteria and protozoans, live in or on birds and can travel along with

344 them. The most known examples are emergent infectious diseases such as avian

345 Influenza and West Nile Virus [24], but other microorganisms can be dispersed in

346 association with other propagules dispersed by birds, including diaspore parasites [74]

347 and viruses and bacteria associated to ectoparasites (e.g. Lyme disease in ticks [22]).

348 The spores of fungi [75], as well as the diaspores of many plant taxa, including

349 bryophytes [30], ferns [2], conifers (e.g. [76]) and both aquatic and land angiosperms

350 (e.g. $[23,51])$ are also frequently dispersed by birds. Among invertebrates, we highlight

351 ectoparasites (e.g. fleas and ticks; e.g. [22]), land [77] and aquatic [78] snails, and

352 aquatic microinvertebrates such as rotifers and crustaceans, but other invertebrates such

353 as flies, hemipterans and other arthropods, as well as nematodes and other worms, can

354 also be dispersed occasionally by birds (e.g. [79]). Birds disperse all these organisms as

355 dormant propagules (e.g. plant seeds, invertebrate cysts and resting eggs), fragments

356 (typically for plants) and/or whole individuals (e.g. snails attached to feet and/or

357 plumage, pathogens and parasites travelling with or within the vector). Vectored

358 dispersal can be triggered by (1) the intentional lure provided by an associated reward,

359 such as the pulp consumed by frugivores, (2) a predation event, in which a fraction of

360 the propagules survives gut passage (e.g. granivory), (3) involuntary ingestion, such as 
361 the consumption of seeds and cysts by filter-feeding birds, (4) attachment of propagules

362 to the vector's body (e.g. to the bird's feet or feathers), or (5) the transmission of

363 pathogens or parasites. Some of the mentioned organisms are known to use bird-

364 mediated LDD services, including plants, invertebrates (mainly zooplankton) and

365 parasites (see main text), but empirical evidence is scarce for the vast majority.

366 Vectored dispersal generally occurs over small spatial scales. Plants, for

367 example, are rarely dispersed over more than 1,500 m [65]. However,

368 LDD operates beyond the scale of a local population, ranging from the landscape scale

369 (at which LDD links metapopulations and metacommunities) to the regional and

370 biogeographical scales (at which LDD leads to the colonization of distant and remote

371 areas). In Figure I we provide some examples of vectored LDD operating at different

372 spatial scales.

\section{Box 2. Bird migration patterns}

375 Migration is a directional movement between separate breeding and wintering areas.

376 Birds undertake extraordinary migratory journeys, crossing hundreds or thousands of

377 kilometers, often over entire continents or between them. Migration consists, in most

378 cases, of a series of consecutive long-distance flights interspersed with stopover periods

379 for resting and feeding (but see [80] for extreme, non-stop flights of waders across the

380 entire Pacific ocean). The distance and frequency of non-stop migratory flights (Figure

381 I), which set the potential for propagule LDD, are the result of species-specific

382 migration strategies, defined according to a trade-off between time, energy and safety

383 [42, 43]. During migration, birds spend most of their time feeding and resting at

384 stopover sites, thus generating local-scale dispersal. In contrast, migratory flights can be

385 expected to promote less frequent, long distance dispersal events. If propagules are 
retained long enough, birds can transport them over hundreds of kilometers - and occasionally over more than one thousand kilometers (Figure I).

data, or estimated using theoretical calculations based on aerodynamic theory (Box 4).

390 Maximum migratory distances calculated from empirical data are shorter than those

391 derived from theoretical calculations, which probably reflects the influence of

392 individual strategies and external factors such as landscape configuration (e.g.

393 movement barriers). Despite the rapid increase in the use of satellite-based tracking

394 technologies, detailed movement data are still lacking for a large proportion of bird

395 species, in particular smaller species such as passerines. This means that detailed

396 knowledge of migratory routes and connectivity is still lacking for most bird species,

397 especially high-frequency data obtained at large spatio-temporal scales. We expect

398 technological advances in animal tracking (already under development and test) to

399 improve our knowledge in a near future, namely through the production of smaller and

400 lighter satellite tags [81]. It will allow a deeper mechanistic understanding of the

401 processes determining flight performance in migrating birds, which in turn will promote

402 the refinement of mechanistic models (e.g. Box 4).

403

404 Box 3. Propagule retention time

405 Propagule retention time is often considered to be the most important determinant of

406 dispersal kernels $[11,82]$, yet the morphological traits, physiological processes and

407 environmental factors behind its intra- and inter-specific variation are still poorly

408 understood. For ingested propagules, the range of gut retention times (GRT) varies

409 greatly among taxa: in passerines GRT peaks at 20 to 60 minutes [54] and show

410 distribution tails that do not extend beyond a few hours, whereas in waterbirds GRT 
411 peaks at 1 to 11 hours and show long tails reaching 72 hours (e.g. [83, 84]; Figure I).

412 GRT scales positively with body mass in passerines [85] but negatively in waterbirds

413 [11]. These contrasting relationships might be related to a trade-off between GRT

414 (larger birds have longer guts through which propagules take longer to pass) and

415 propagule survival (larger birds have stronger gizzards that destroy a higher proportion

416 of propagules that spend longer periods within them), though further research is still

417 needed. For externally-attached propagules, the only study that measured attachment

418 time to bird feathers showed an exponential decrease of retention time up to a maximum

419 of nine hours, strongly associated with preening and ruffling rates [86]; and for

420 pathogens, the duration of infection (i.e., retention time) is variable. For example, the

421 duration of infection by West Nile virus in various bird orders and by Influenza A in

422 mallards peaks at approx. 3 days, extending up to 7 and 34 days, respectively $[87,88]$.

423 Other endoparasites (e.g. Plasmodium) and ectoparasites (e.g. ticks) cause life-lasting

424 infections in birds.

425 It is also worth noting that propagule retention and flying activity might

426 influence each other, but we still lack a methodology to measure retention time while

427 birds are flying. A study on the effect of physical activity (swimming) on seed retention

428 time using mallards showed enhanced propagule survival but slightly shorter retention

429 times at higher physical activity [89]. On the other hand, travelling with the extra

430 weight of a large (ingested) propagule load might affect flying performance [90].

431 Parasites and pathogens might also affect the birds' physical condition and migratory

432 performance, such as in swans infected by Influenza, which delayed the start of their

433 migratory flights for more than a month, until the end of the infectious period [91] - but

434 not in two passerine vectors (Swainson's thrush and gray catbird) experimentally

435 infected with West-Nile Virus, whose migratory activity was unaffected [92]. 
437 Box 4. Allometric scaling

438 The size of organisms is an important determinant of many vital physiological and

439 behavioral processes [93]. Hence, body mass (M) is often related to many

440 morphological and functional traits (Y) by this general expression, where $\mathrm{b}$ is the

441 scaling exponent [93]:

$442 \mathrm{Y}=\mathrm{Y}_{0} \mathrm{M}^{\mathrm{b}}$

443 Let $U$ be the flight speed and $R$ the propagule retention time. Dispersal distance (D) can 444 be estimated as:

$445 \quad \mathrm{D}=\mathrm{c} \mathrm{UR}$

446 where $\mathrm{c}$ is a correction factor for departures from the assumption of linear movement at 447 constant speed from propagule uptake to release (adapted from [94]). U scales to the 448 body mass of animal vectors [94]:

$449 \mathrm{U}=15.9 \mathrm{M}^{0.13}$

450 For internal dispersal, $\mathrm{R}$ scales also to the body mass of the animal ingesting the 451 propagule, so that:

$452 \quad \mathrm{R}=\mathrm{R}_{0} \mathrm{M}^{\mathrm{b}}$

453 where $\mathrm{R}_{0}$ and $\mathrm{b}$ take different values for different functional groups (e.g. passerines vs.

454 waterfowl $[11,85])$.

455 These formulae provide a rough estimate of the maximal (or potential) dispersal 456 distance, assuming that the vectoring animal keeps on moving until the propagule is 457 released. But for dispersal to be effective in most cases, the vector must land before the 458 propagule is released, i.e. the flight time (T) must be equal or shorter than the retention 459 time $(\mathrm{T} \leq \mathrm{R})$. We can estimate flight time according to the equation:

$460 \quad \mathrm{~T}=\mathrm{k}^{-1} \ln (1+\mathrm{f})$ 
461 where $\mathrm{K}$ is the rate of mass loss and $\mathrm{f}$ is the relative fuel load. The flight distance $(\mathrm{Y})$ is

462 the multiplication of the flight time by the flight speed [43]:

$463 \quad \mathrm{Y}=\mathrm{U} \mathrm{k}^{-1} \ln (1+\mathrm{f})$

464 Flight time and distance can be expected to scale with body mass, as $\mathrm{k}$ is inversely

465 related to metabolic power consumption during flight (P). P shows the following

466 empirical relationship with body mass [95]:

$467 \quad \mathrm{P}=53.65 \mathrm{M}^{0.74}$

468 whose exponent is higher for calculations based on the aerodynamic theory [96], where:

$469 \mathrm{P}=44.05 \mathrm{M}^{0.975}$

470 These calculations have a number of limitations. Firstly, they are based on the

471 conservative assumption that only fat, rather than fat and protein, is burned during the

472 migratory flight. Second, they focus on estimating maximum (i.e. potential) flight time

473 and distance, which might not be good indicators of the overall migration strategy.

474 Instead, mode migratory distances might be obtained by using usual, rather than

475 maximum, fat loads. In this sense, it is important to note that maximum dispersal

476 distances set the potential limit for one-step LDD (Figure I), even though mode

477 distances (which are far more frequent) are often large enough to result in LDD.

478

479 Outstanding Questions

480

481 Dispersal ecology

482 - What characteristics (besides body mass) determine the vectoring capacity of birds

483 during migration?

484 - Can allometric scaling be used to estimate multi-vector dispersal kernels? 
485 - How flying activity, particularly during migration, modifies propagule retention time?

486 Experiments measuring propagule retention time of birds flying on wind tunnels can

487 provide a solution to this question.

488 - How many propagules are dispersed by migratory birds each year and at which scale?

489 I.e., how strong is the propagule pressure generated by migratory birds at different

490 spatial scales? Can major stopover areas where migrating birds congregate function as

491 hotspots for propagule deposition?

492

493 Ecological consequences

494 - What is the colonization success of species and individuals dispersed by migratory

495 birds? Can deposition hotspots (such as major stopover areas) promote colonization and

496 maintain or boost regional diversity?

497 - Does LDD mediated by migratory birds influence metapopulation and

498 metacommunity dynamics, particularly in fragmented habitats? Will the observed

499 declines in migratory bird populations reduce the connectivity between populations?

500 - Can the dispersal services provided by migratory birds determine phylo- and bio-

501 geographic patterns?

502 - To what extent can the vectoring role of migratory birds accelerate the rate of range

503 expansion and shifts? Will it suffice to compensate for the impact of climate change?

504 - What is the role of migrating birds as mobile linkers among ecosystems, particularly

505 as providers of ecosystem services?

506

507 Conservation biology

508 - Which types of invasive species can be (regularly) dispersed by migratory birds? 
509 - Can migratory birds accelerate the spread of pathogens? What characteristics of

510 pathogens favour their dispersal?

511 - Can species distribution models, particularly those used to predict range adjustments

512 and design conservation strategies, incorporate predictable LDD estimates?

\section{Glossary}

515 Endozoochory: dispersal of propagules inside an animal vector

516 Epizoochory: dispersal of propagules attached to an animal vector

517 Disjunct distribution: species showing large discontinuities in their distribution (e.g.

518 transoceanic and bipolar distributions).

519 Dispersal kernel: a probability distribution of dispersal distances and the associated

520 spatial distribution of dispersal units.

521 Dispersal vector: any agent transporting propagules (e.g. birds or wind).

522 Long distance dispersal (LDD): dispersal acting beyond local scales, typically across

523 population boundaries.

524 Propagule: a vectored dispersal unit.

525 Range shift: shift in the geographic distribution of species, often in response to 526 environmental change (e.g. climate change).

527 Tail of probability distribution: the range of a given variable (e.g. dispersal distance)

528 that has a disproportionate low occurrence probability, whose length and thickness

529 depend on the distribution kurtosis and skewness. LDD is characterized by right-

530 skewed, leptokurtic distributions (i.e. large distance values occur at low probability).

531

532 Acknowledgements 
533 We thank Ran Nathan and one anonymous reviewer for useful discussions. This study

534 was supported by project CGL2015-65055-P from Ministerio de Economía y

535 Competitividad (Spain) and RECUPERA 2020, Hito 1.1.1, cofinanced by the European

536 Regional Development Fund (FEDER).

537

\section{References}

539 1. De Queiroz, A. (2014) The monkey's voyage: how improbable journeys shaped $540 \quad$ the history of life. Basic Books.

541 2. Gillespie, R.G., et al. (2012) Long-distance dispersal: a framework for $542 \quad$ hypothesis testing. Trends Ecol. Evol. 27, 47-56.

543 3. Nathan, R. and Nathan, O. (2014) Unlikely yet pivotal long dispersals. Science $544 \quad 344,153-154$.

545 4. Nathan, R., et al. (2008) Mechanisms of long-distance seed dispersal. Trends $546 \quad$ Ecol. Evol. 23, 638-647.

547 5. Anderson, J.T., et al. (2011) Extremely long-distance seed dispersal by an $548 \quad$ overfished Amazonian frugivore. Proc. R. Soc. B, rspb20110155.

549 6. Campos-Arceiz, A., et al. (2008) Behaviour rather than diet mediates seasonal 550 differences in seed dispersal by Asian elephants. Ecology 89, 2684-2691.

551 7. Vellend, M., et al. (2003) Dispersal of Trillium seeds by deer: implications for 552 long-distance migration of forest herbs. Ecology 84, 1067-1072.

553 8. Holbrook, K.M., et al. (2002) Implications of long-distance movements of 554 frugivorous rain forest hornbills. Ecography 25, 745-749.

555 9. Corlett, R.T. (2009) Seed dispersal distances and plant migration potential in 556 tropical East Asia. Biotropica 41, 592-598. 
557 10. Tsoar, A., et al. (2011) A movement ecology approach to study seed dispersal

558

559

560

561

562

563

564

565

566

567

568

569

570

571

572

573

574

575

576

577

578

579

580

581 and plant invasion: an overview and application of seed dispersal by fruit bats. In Fifty years of invasion ecology: the legacy of Charles Elton (Richardson, D.M., ed), pp. 103-119, Wiley-Blackwell.

11. Viana, D.S., et al. (2013) Allometric scaling of long-distance seed dispersal by migratory birds. Am. Nat. 181, 649-662.

12. Elith*, J., et al. (2006) Novel methods improve prediction of species' distributions from occurrence data. Ecography 29, 129-151.

13. Phillips, S.J., et al. (2006) Maximum entropy modeling of species geographic distributions. Ecol. Model. 190, 231-259.

14. Mummenhoff, K. and Franzke, A. (2007) Gone with the bird: Late tertiary and quaternary intercontinental long-distance dispersal and allopolyploidization in plants. Syst. Biodivers. 5, 255-260.

15. Le Roux, J.J., et al. (2014) Relatedness defies biogeography: the tale of two island endemics (Acacia heterophylla and A. koa). New Phytol. 204, 230-242.

16. Popp, M., et al. (2011) A single Mid-Pleistocene long-distance dispersal by a bird can explain the extreme bipolar disjunction in crowberries (Empetrum). Proc. Natl. Acad. Sci. USA 108, 6520-6525.

17. Lewis, L.R., et al. (2014) Direct long-distance dispersal shapes a New World amphitropical disjunction in the dispersal-limited dung moss Tetraplodon (Bryopsida: Splachnaceae). J. Biogeogr. 41, 2385-2395.

18. Piñeiro, R., et al. (2012) Circumarctic dispersal and long-distance colonization of South America: the moss genus Cinclidium. J. Biogeogr. 39, 2041-2051.

19. Figuerola, J., et al. (2005) Invertebrate eggs can fly: evidence of waterfowlmediated gene flow in aquatic invertebrates. Am. Nat. 165, 274-280. 
582 20. Muñoz, J., et al. (2013) Bird migratory flyways influence the phylogeography of the invasive brine shrimp Artemia franciscana in its native American range.

584 PeerJ 1, e200.

585

21. Miura, O., et al. (2011) Flying shells: historical dispersal of marine snails across

586 Central America. Proc. R. Soc. B 282.

587 22. Smith, R.P., et al. (1996) Role of bird migration in the long-distance dispersal of 588 Ixodes dammini, the vector of Lyme disease. J. Infect. Dis. 174, 221-224.

23. Stiles, F.G. (2000) Animals as seed dispersers. In Seeds: the ecology of regeneration in plant communities (Fenner, M., ed), pp. 111-124, CABI Publishing.

592 24. Altizer, S., et al. (2011) Animal migration and infectious disease risk. Science 593 $331,296-302$.

594

25. Fenton, A., et al. (2002) Parasite transmission: reconciling theory and reality. $J$. Anim. Ecol. 71, 893-905.

26. Somveille, M., et al. (2013) Mapping global diversity patterns for migratory birds. PLOS ONE 8, e70907.

598 27. Chesser, R.T. and Levey, D.J. (1998) Austral migrants and the evolution of 599 migration in New World birds: diet, habitat, and migration revisited. Am. Nat.

600 $152,311-319$.

28. Conklin, J.R., et al. (2010) Breeding latitude drives individual schedules in a 602 trans-hemispheric migrant bird. Nat. Commun. 1, 67.

603 29. Viana, D.S., et al. (2016) Overseas seed dispersal by migratory birds. Proc. R. 604 Soc. B 283.

605 30. Lewis, L.R., et al. (2014) First evidence of bryophyte diaspores in the plumage 606 of transequatorial migrant birds. PeerJ 2, e424. 
607 31. Nogales, M., et al. (2012) Evidence for overlooked mechanisms of long-distance 608 seed dispersal to and between oceanic islands. New Phytol. 194, 313-317.

609

32. Fridriksson, S. (1975) Surtsey: evolution of life on a volcanic island.

610 Butterworths.

611 33. Bucher, E.H. and Bocco, P.J. (2009) Reassessing the importance of granivorous pigeons as massive, long-distance seed dispersers. Ecology 90, 2321-2327.

613 34. Holland, R.A., et al. (2009) The secret life of oilbirds: new insights into the movement ecology of a unique avian frugivore. PLoS ONE 4, e8264.

615

35. Mokotjomela, T.M., et al. (2013) Potential seed dispersal distances of native and 616 non-native fleshy fruiting shrubs in the South African Mediterranean climate region. Plant Ecol. 214, 1127-1137.

618

36. Raulings, E., et al. (2011) Do birds of a feather disperse plants together? Freshwat. Biol. 56, 1390-1402.

620 37. Lebarbenchon, C., et al. (2009) Spread of avian Influenza viruses by common 621 teal (Anas crecca) in Europe. PLoS ONE 4, e7289.

622 38. Nathan, R., et al. (2008) A movement ecology paradigm for unifying organismal 623 movement research. Proc. Natl. Acad. Sci. USA 105, 19052-19059.

624

39. Burns, K.C. (2002) Seed dispersal facilitation and geographic consistency in 625 bird-fruit abundance patterns. Global Ecol. Biogeogr. 11, 253-259.

626 40. Figuerola, J., et al. (2003) Passive internal transport of aquatic organisms by 627 waterfowl in Doñana, south-west Spain. Global Ecol. Biogeogr. 12, 427-436. 628 41. Wright, S.J., et al. (2008) Understanding strategies for seed dispersal by wind 629 under contrasting atmospheric conditions. Proc. Natl. Acad. Sci. USA 105, 630 19084-19089. 
631 42. Alerstam, T. (2011) Optimal bird migration revisited. Journal of Ornithology

$632 \quad 152,5-23$.

633 43. Hedenström, A. (2008) Adaptations to migration in birds: behavioural strategies, 634 morphology and scaling effects. Phil. Trans. R. Soc. B 363, 287-299.

635 44. Viana, D.S., et al. (2013) Migratory strategies of waterbirds shape the 636 continental-scale dispersal of aquatic organisms. Ecography 36, 430-438.

637 45. Soons, M.B., et al. (2008) Small seed size increases the potential for dispersal of 638 wetland plants by ducks. J. Ecol. 96, 619-627.

639 46. Sorensen, A.E. (1986) Seed dispersal by adhesion. Annu. Rev. Ecol. Syst. 17, $640 \quad 443-463$.

641 47. McCabe, J.D. and Olsen, B.J. (2015) Landscape-scale habitat availability, and 642 not local geography, predicts migratory landbird stopover across the Gulf of Maine. J. Avian Biol. 46, 395-405.

644 48. Shamoun-Baranes, J., et al. (2010) Stochastic atmospheric assistance and the use 645 of emergency staging sites by migrants. Proc. R. Soc. B 277, 1505-1511.

646 49. Caughlin, T.T., et al. (2014) The importance of long-distance seed dispersal for 647 the demography and distribution of a canopy tree species. Ecology 95, 952-962.

648 50. Schupp, E.W., et al. (2010) Seed dispersal effectiveness revisited: a conceptual 649 review. New Phytol. 188, 333-353.

650 51. Van Leeuwen, C.H.A., et al. (2012) Gut travellers: internal dispersal of aquatic 651 organisms by waterfowl. J. Biogeogr. 39, 2031-2040.

652 52. Costa, J.M., et al. (2014) Endozoochory largely outweighs epizoochory in 653 migrating passerines. J. Avian Biol. 45, 59-64.

654 53. Herrera, C.M. (1984) A study of avian frugivores, bird-dispersed plants, and 655 their interaction in Mediterranean scrublands. Ecol. Monogr. 54, 1-23. 
656 54. Traveset, A. (1998) Effect of seed passage through vertebrate frugivores' guts on germination: a review. Perspect. Plant Ecol. Evol. Syst. 1, 151-190.

658 55. Nee, S., et al. (1991) The relationship between abundance and body size in British birds. Nature 351, 312-313.

660 56. Santamaría, L. (2002) Why are most aquatic plants widely distributed? Dispersal, clonal growth and small-scale heterogeneity in a stressful environment. Acta Oecol. 23, 137-154.

663 57. De Meester, L., et al. (2002) The Monopolization Hypothesis and the dispersal664 gene flow paradox in aquatic organisms. Acta Oecol. 23, 121-135.

665 58. Carlo, T.A. and Tewksbury, J.J. (2014) Directness and tempo of avian seed 666 667 $102,248-255$.

668

59. Amezaga, J.M., et al. (2002) Biotic wetland connectivity-supporting a new approach for wetland policy. Acta Oecol. 23, 213-222.

670 60. Levey, D.J., et al. (2008) Modelling long-distance seed dispersal in heterogeneous landscapes. J. Ecol. 96, 599-608.

672 61. Yu, S., et al. (2014) Species diversity and distribution of Ruppia in China: Potential roles of long-distance dispersal and environmental factors. $J$. Syst. Evol. 52, 231-239.

675 62. Heleno, R. and Vargas, P. (2015) How do islands become green? Global Ecol. 676 Biogeogr. 24, 518-526.

677 63. Green, A.J. (2015) The importance of waterbirds as an overlooked pathway of 678 invasion for alien species. Divers. Distrib. 22, 239-247.

679 64. Trakhtenbrot, A., et al. (2005) The importance of long-distance dispersal in 680 biodiversity conservation. Divers. Distrib. 11, 173-181. 
681 65. Corlett, R.T. and Westcott, D.A. (2013) Will plant movements keep up with $682 \quad$ climate change? Trends Ecol. Evol. 28, 482-488.

683 66. Urban, M.C. (2015) Accelerating extinction risk from climate change. Science $684 \quad 348,571-573$.

685 67. Bocedi, G., et al. (2014) RangeShifter: a platform for modelling spatial eco686 evolutionary dynamics and species' responses to environmental changes. $687 \quad$ Methods Ecol. Evol. 5, 388-396.

688 68. Cunze, S., et al. (2013) Are plant species able to keep pace with the rapidly 689 changing climate? PLoS ONE 8, e67909.

690 69. Wang, B.C. and Smith, T.B. (2002) Closing the seed dispersal loop. Trends $691 \quad$ Ecol. Evol. 17, 379-386.

692 70. Hampe, A., et al. (2003) Rangewide phylogeography of a bird-dispersed 693 Eurasian shrub: contrasting Mediterranean and temperate glacial refugia. Mol. 694 Ecol. 12, 3415-3426.

695 71. King, R.A., et al. (2002) Population differentiation of Potamogeton pectinatus 696 in the Baltic Sea with reference to waterfowl dispersal. Mol. Ecol. 11, 1947$697 \quad 1956$.

698 72. Brochet, A.-L., et al. (2009) The role of migratory ducks in the long-distance 699 dispersal of native plants and the spread of exotic plants in Europe. Ecography 700 $32,919-928$.

701 73. Jønsson, K.A., et al. (2016) Tracking animal dispersal: from individual 702 movement to community assembly and global range dynamics. Trends Ecol. Evol. 31, 204-214.

704 74. Del Rio, C.M., et al. (1996) Seed dispersers as disease vectors: bird transmission 705 of mistletoe seeds to plant hosts. Ecology, 912-921. 
706 75. Malloch, D. and Blackwell, M. (1992) Dispersal of fungal diaspores. In The fungal community: its organization and role in the ecosystem (Dighton, J., et al., eds), pp. 147-171, Marcel Dekker Inc.

709 76. Tomback, D.F. and Linhart, Y.B. (1990) The evolution of bird-dispersed pines. Evol. Ecol. 4, 185-219.

711 77. Wada, S., et al. (2012) Snails can survive passage through a bird's digestive system. J. Biogeogr. 39, 69-73.

78. van Leeuwen, C.H.A., et al. (2012) Experimental quantification of long distance dispersal potential of aquatic snails in the gut of migratory birds. PLoS ONE 7,

79. Frisch, D., et al. (2007) High dispersal capacity of a broad spectrum of aquatic invertebrates via waterbirds. Aquat. Sci. 69, 568-574.

718 80. Gill, R.E., et al. (2009) Extreme endurance flights by landbirds crossing the Pacific Ocean: ecological corridor rather than barrier? Proc. R. Soc. B 276, 447457.

721 81. Kays, R., et al. (2015) Terrestrial animal tracking as an eye on life and planet. Science 348, aaa2478.

723 82. Guttal, V., et al. (2011) Retention time variability as a mechanism for animal mediated long-distance dispersal. PLoS ONE 6, e28447.

725 83. Charalambidou, I., et al. (2005) Digestive plasticity in mallard ducks modulates dispersal probabilities of aquatic plants and crustaceans. Funct. Ecol. 19, 513519.

728 84. Charalambidou, I., et al. (2003) Effect of ingestion by five avian dispersers on 729 the retention time, retrieval and germination of Ruppia maritima seeds. Funct. Ecol. 17, 747-753. 
731 85. Karasov, W.H. (1990) Digestion in birds: chemical and physiological determinants and ecological implications. Stud. Avian Biol. 13, 391-415.

733 86. Yumoto, T. (1999) Seed dispersal by Salvin's curassow, Mitu salvini (Cracidae), 734 in a tropical forest of Colombia: direct measurements of dispersal distance. Biotropica 31, 654-660.

736 87. Komar, N., et al. (2003) Experimental infection of North American birds with 737 the New York 1999 strain of West Nile virus. Emerging Infect. Dis. 9, 311-322.

738 88. Latorre-Margalef, N., et al. (2009) Effects of Influenza A virus infection on 739 migrating mallard ducks. Proc. R. Soc. B 276, 1029-1036.

740 89. Kleyheeg, E., et al. (2015) Bird-mediated seed dispersal: reduced digestive efficiency in active birds modulates the dispersal capacity of plant seeds. Oikos $124,899-907$.

743 90. Piersma, T. and Gill, R.E., Jr. (1998) Guts don't fly: small digestive organs in 744 obese bar-tailed godwits. Auk 115, 196-203.

745 91. Van Gils, J.A., et al. (2007) Hampered foraging and migratory performance in swans infected with low-pathogenic avian Influenza A virus. PLoS ONE 2, e184.

748 92. Owen, J., et al. (2006) Migrating birds as dispersal vehicles for West Nile virus. EcoHealth 3, 79-85.

750 93. West, G.B., et al. (1997) A general model for the origin of allometric scaling laws in biology. Science 276, 122-126.

752 94. Schurr, F.M., et al. (2009) Long-Distance Seed Dispersal. In Annual plant reviews: fruit development and seed dispersal, pp. 204-237, Wiley-Blackwell.

754 95. Hedenström, A. (2010) Extreme endurance migration: what is the limit to nonstop flight? PLoS Biol. 8, e1000362. 
756 96. McWilliams, S.R., et al. (2004) Flying, fasting, and feeding in birds during

757 migration: a nutritional and physiological ecology perspective. J. Avian Biol. 35, $758 \quad 377-393$.

759 97. Hijmans, R.J., et al. (2005) Very high resolution interpolated climate surfaces 760 for global land areas. Int. J. Climatol. 25, 1965-1978.

761 98. Hall-Karlsson, K.S.S. and Fransson, T. (2008) How far do birds fly during one 762 migratory flight stage? Ringing Migr. 24, 95-100.

763 99. Hedenström, A. and Alerstam, T. (1992) Climbing performance of migrating 764 birds as a basis for estimating limits for fuel-carrying capacity and muscle work. J. Exp. Biol. 164, 19-38.

766 100. Jenni, L. and Jenni-Eiermann, S. (1998) Fuel supply and metabolic constraints 767 in migrating birds. J. Avian Biol. 29, 521-528. 
770 Figure 1. Movement ecology framework for propagules dispersed by migratory birds.

771 Note that, independently of propagule adaptations to its vectors and thus to movement,

772 propagule movement relies on the vector movement as its key external factor, and thus

773 the vectored organisms' movement is nested within the vectors' movement (see [10]).

774

775 Figure 2. Estimation of rapid range shifts mediated by migratory birds: example of a

776 population present in Doñana National Park, Spain, dispersed by a waterfowl species

777 weighing $300 \mathrm{~g}$ (orange line in the dispersal kernel) and migrating along a route (orange

778 polygon) within the Palaearctic-African flyway. The grey line corresponds to a

779 waterfowl species weighing $1 \mathrm{~kg}$ (for comparison purposes). Dispersal kernels were

780 parameterized according to empirical data and estimated according to a mechanistic

781 model [11], where LDD was considered as dispersal mediated by bird migratory flights,

782 i.e. flights $>100 \mathrm{~km}$. Habitat suitability was assumed to be within the range $10-25^{\circ} \mathrm{C}$ of

783 maximum March temperature (note that this is only an example; temperature

784 information was obtained from [97]). The probability of arrival and establishment in a

785 suitable location corresponds to (1 - cumulative distance frequency) (grey scale

786 corresponding to the dispersal kernel above).

788 Figure I (Box 1). Examples of vectored LDD operating at different spatial scales: (A)

789 ticks and Lyme disease dispersed by migratory landbirds over $37 \mathrm{~km}$ [22], (B)

790 macrophyte seeds and zooplankton eggs dispersed by migratory waterfowl over

791 distances ranging from tens to hundreds of kilometers [11], (C) terrestrial plant seeds

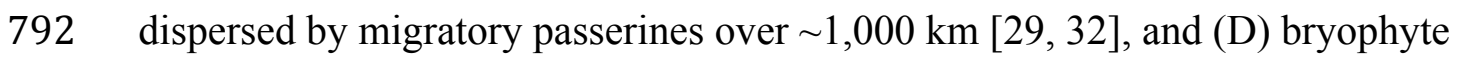


793 diaspores dispersed by transequatorial migrant shorebirds over distances up to 15,000

$794 \mathrm{~km}[30]$. Solid and dashed arrows correspond to examples of dispersal events either

795 directly observed or supported by compelling evidence, respectively.

796

797 Figure I (Box 2). Frequency distribution of migratory distances for waterfowl

798 (Anatidae; A; data from [11]) and passerines (mostly frugivores; B; data read from

799 [98]). Distances were obtained from ringing data by measuring the distance between

800 two consecutive sightings within a period of six (A) or seven (B) days. Within these

801 time periods, most waterfowl make only a single migratory movement (see [44] for

802 details); passerines, nevertheless, can make more than one migratory flight. Distances

$803<50 \mathrm{~km}$ were excluded.

804

805 Figure I (Box 3). Probability distribution of gut retention times. (A) Waterfowl:

806 lognormal distribution fitted to aggregated experimental raw-data (individual gut

807 retention times of plant seeds fed to seven duck species [11]). (B) Passerines: lognormal

808 distribution fitted to summarized experimental data (mean and standard deviation of the

809 gut retention time of inert tracers fed to 13 passerine species [82]). The dashed line

810 represents retention times beyond the standard deviation.

811

812 Figure I (Box 4). Maximum range distances of bird migratory flights as a function of

813 body mass, calculated according to empirical (A) and allometric (B) relationships.

814 Allometric relationships were based on the bird's maximum fuel-loading capacity

$815(\mathrm{hmax}=1.42$ mass $-0.0554 ;$ [99]). Maximum fuel loads (fmax) were estimated as

816 hmax-1, and power consumption was transformed into mass loss by converting $37.6 \mathrm{~kJ}$

817 into one gram of fat (assuming that only fat is burned; [100]). 
818 Figure 1

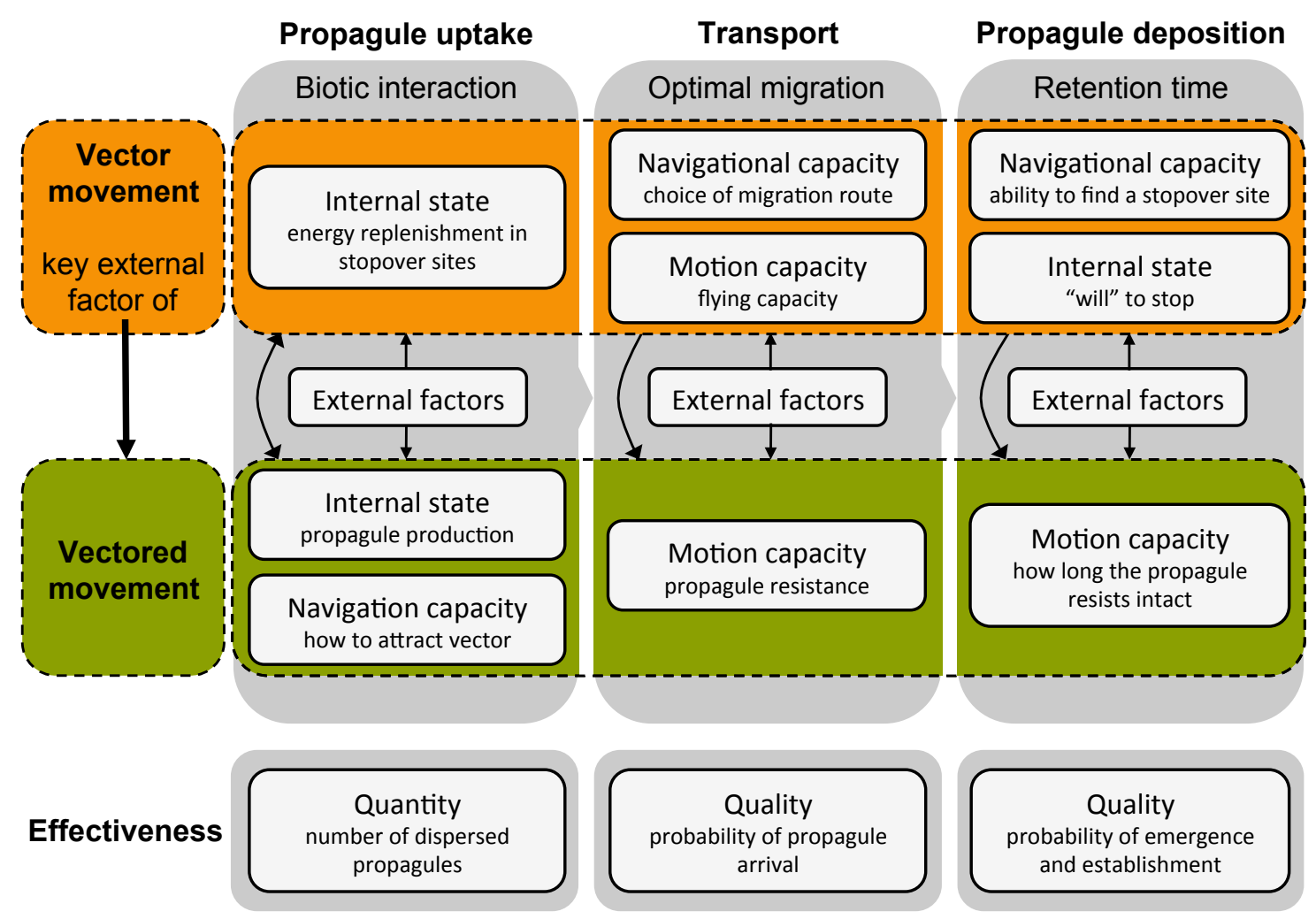


820 Figure 2

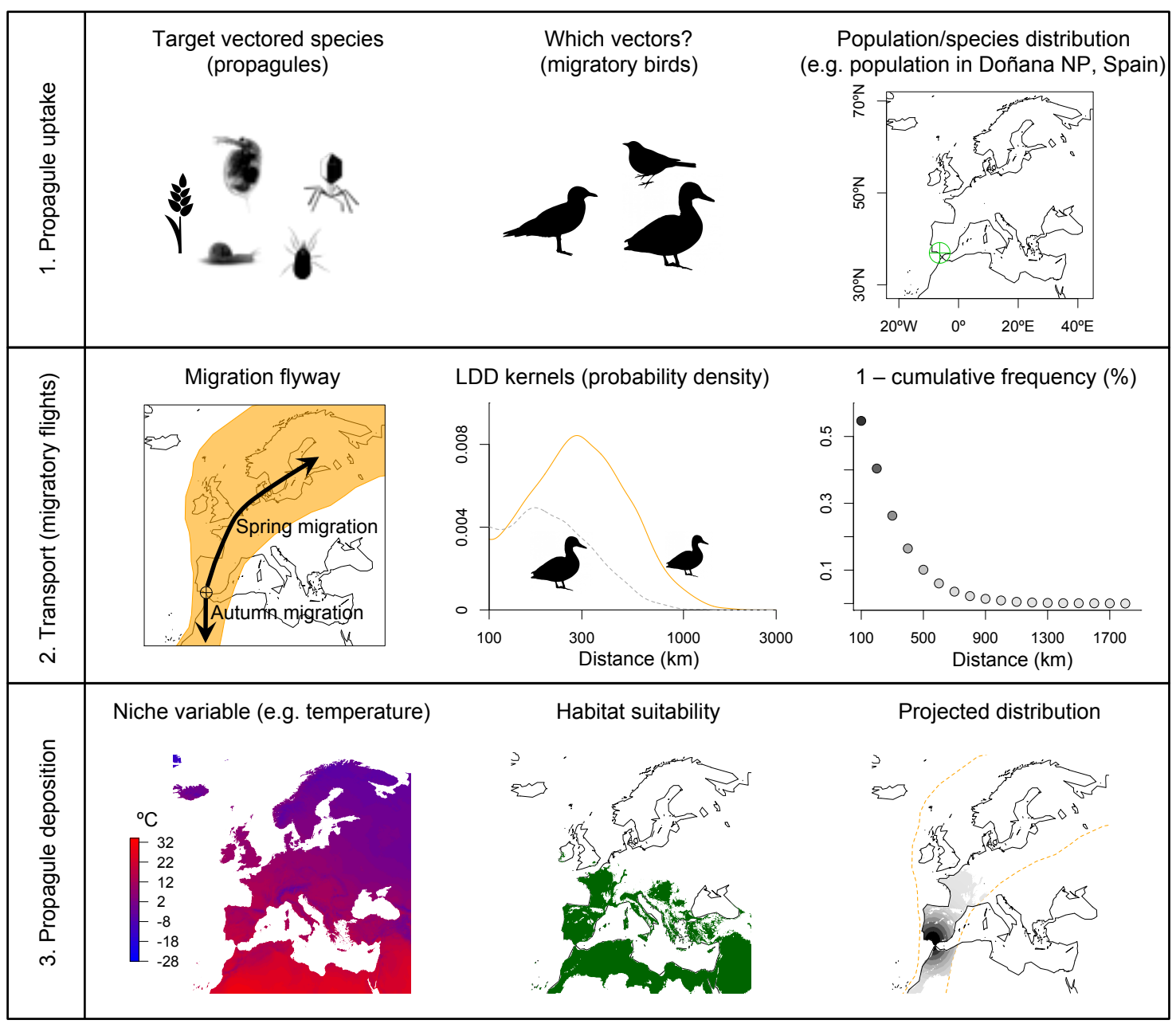

822 
822 Figure I - Box 1

823
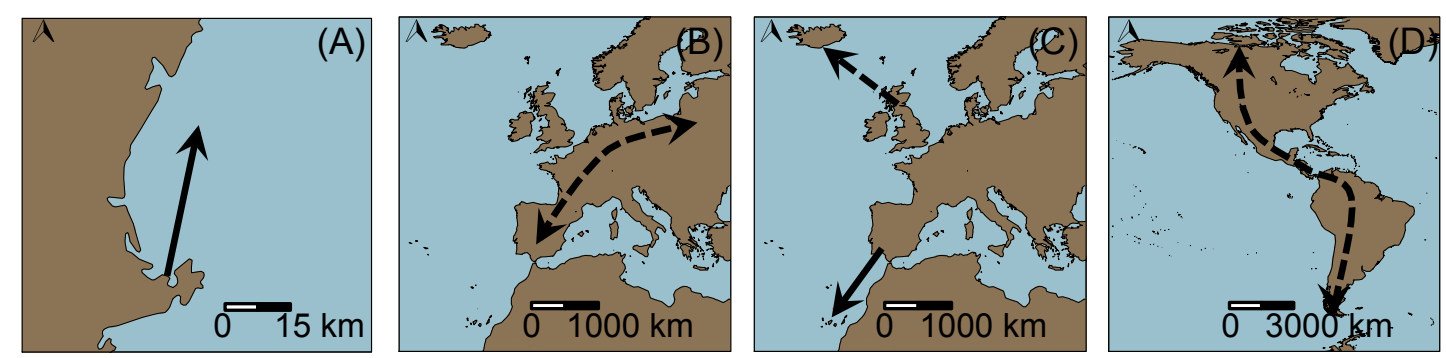

824 
824 Figure I - Box 2

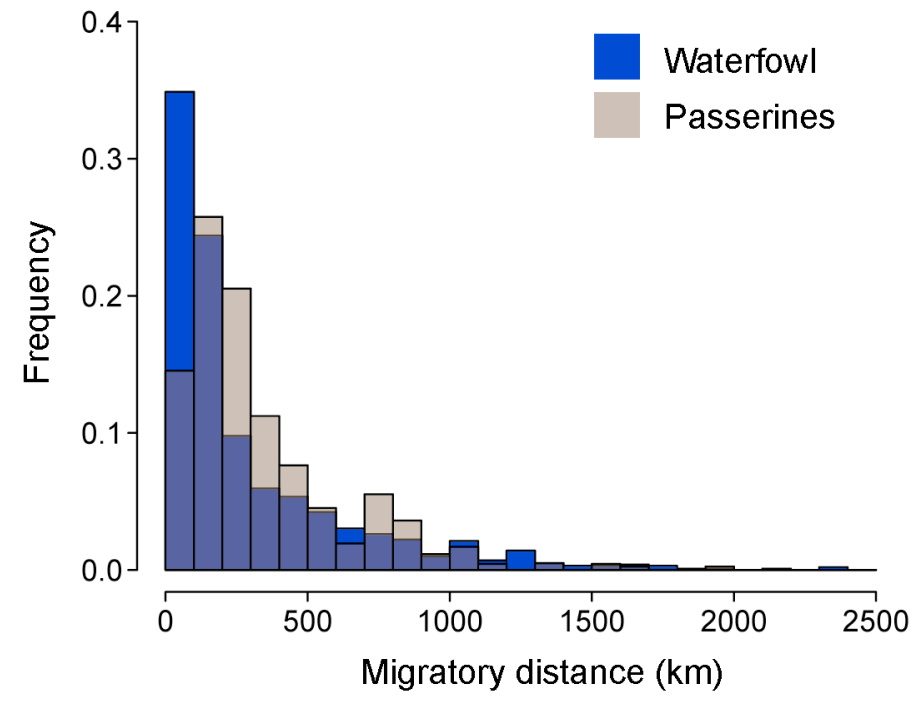

826 
826 Figure I - Box 3
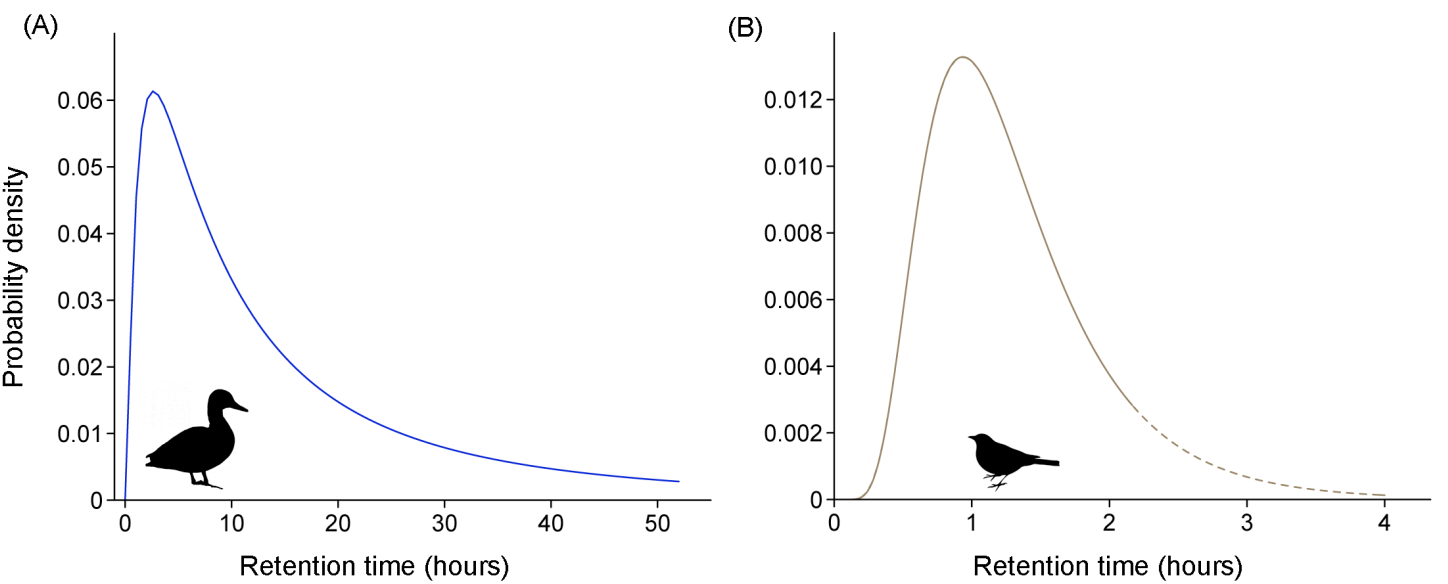

827

828 
828 Figure I - Box 4

(A)

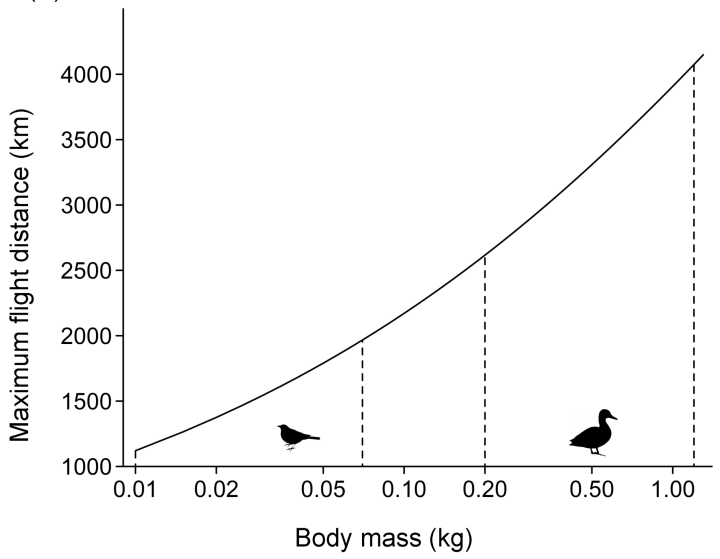

(B)

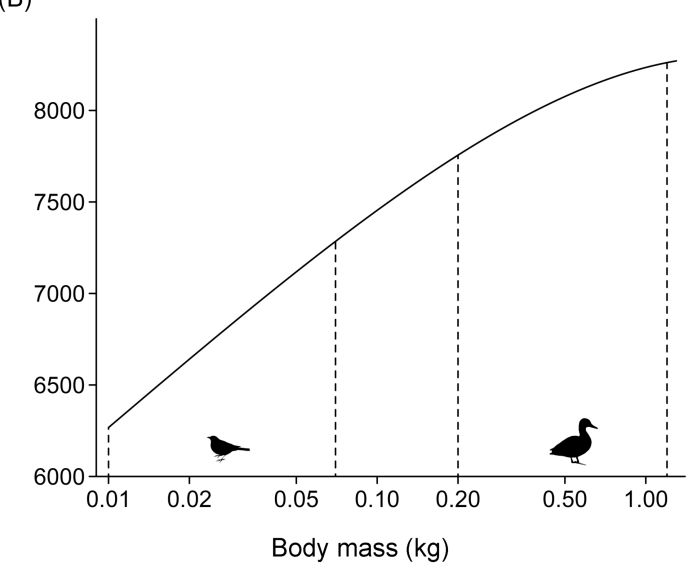

